\title{
A Hydrogen Atmosphere Spectral Model Applied to the Neutron Star X7 in the Globular Cluster 47 Tucanae
}

\section{Citation}

Heinke, Craig 0., George B. Rybicki, Ramesh Narayan, and Jonathan E. Grindlay. 2006. "A Hydrogen Atmosphere Spectral Model Applied to the Neutron Star X7 in the Globular Cluster 47 Tucanae." The Astrophysical Journal 644 (2): 1090-1103. https://doi.org/10.1086/503701.

\section{Permanent link}

http://nrs.harvard.edu/urn-3:HUL.InstRepos:41384972

\section{Terms of Use}

This article was downloaded from Harvard University's DASH repository, and is made available under the terms and conditions applicable to Other Posted Material, as set forth at http:// nrs.harvard.edu/urn-3:HUL.InstRepos:dash.current.terms-of-use\#LAA

\section{Share Your Story}

The Harvard community has made this article openly available. Please share how this access benefits you. Submit a story. 
DRAFT VERSION JULY 12, 2018

Preprint typeset using $\mathrm{LT}_{\mathrm{E}} \mathrm{X}$ style emulateapj v. 6/22/04

\title{
A HYDROGEN ATMOSPHERE SPECTRAL MODEL APPLIED TO THE NEUTRON STAR X7 IN THE GLOBULAR CLUSTER 47 TUCANAE
}

\author{
Craig O. Heinke ${ }^{1,2}$, George B. Rybicki, Ramesh NARAyan, Jonathan E. GRindlay \\ Harvard-Smithsonian Center for Astrophysics, 60 Garden Street, Cambridge, MA 02138; grybicki@cfa.harvard.edu, rnarayan@cfa.harvard.edu, \\ jgrindlay@cfa.harvard.edu \\ Draft version July 12, 2018
}

\begin{abstract}
Current X-ray missions are providing high-quality X-ray spectra from neutron stars (NSs) in quiescent lowmass X-ray binaries (qLMXBs). This has motivated us to calculate new hydrogen-atmosphere models, including opacity due to free-free absorption and Thomson scattering, thermal electron conduction, and selfirradiation by photons from the compact object. We have constructed a self-consistent grid of neutron star models covering a wide range of surface gravities as well as effective temperatures, which we make available to the scientific community.

We present multi-epoch Chandra X-ray observations of the qLMXB X7 in the globular cluster 47 Tuc, which is remarkably nonvariable on timescales from minutes to years. Its high-quality X-ray spectrum is adequately fit by our hydrogen-atmosphere model without any hard power-law component or narrow spectral features. If a mass of $1.4 M_{\odot}$ is assumed, our spectral fits require that its radius be in the range $R_{\mathrm{ns}}=14.5_{-1.6}^{+1.8} \mathrm{~km}(90 \%$ confidence), larger than expected from currently preferred models of NS interiors. If its radius is assumed to be $10 \mathrm{~km}$, then a mass of $M_{\mathrm{ns}}=2.20_{-0.16}^{+0.03} M_{\odot}$ is required. Using models with the appropriate surface gravity for each value of the mass and radius becomes important for interpretation of the highest quality data.

Subject headings: radiative transfer — binaries : X-rays — globular clusters: individual (NGC 104) — stars: neutron
\end{abstract}

\section{INTRODUCTION}

One of the primary goals of neutron star (NS) studies is to constrain the behavior of matter at high densities by measuring NS masses and radii (Lattimer \& Prakash 2001). Mass measurements of high accuracy for a number of radio pulsars in close binary systems (often with inferred NS companions) are consistent with a range of NS masses between 1.25 and $1.45 M_{\odot}$ (Thorsett \& Chakrabartv 1999). Fundamental constraints on NS interior structure can be achieved by measurement of the gravitational redshift from the NS surface (Cottam et al. 2002). Finally, it should be possible to derive constraints on the radius of NSs from spectral fits to their Xray emission if the temperature, composition of atmosphere, and distance to a NS are known, and the magnetic field is sufficiently weak so as not to affect the opacity, or temperature distribution, on the NS surface. These requirements can be fulfilled for X-ray observations of quiescent low-mass Xray binaries (qLMXBs) containing NSs, particularly those located in globular clusters where the distance is well-known (Brown et al. 1998; Rutledge et al. 2002a). In this paper, we perform the most accurate such test currently possible, using accurate hydrogen atmosphere models constructed specifically for this project, and a long Chandra observation of a globular cluster containing a relatively bright and remarkably constant qLMXB.

Several low-mass X-ray binaries which have been identified during outbursts as accreting NS systems have been observed in quiescence (see Campana et al. 1998; Rutledge et al. 2002b). Their quiescent appearance generally differs from that of quiescent systems containing black holes both in their X-ray luminosity (Garcia et al. 2001) and their observed Xray spectrum (Rutledge et al. 1999; McClintock et al. 2004),

\footnotetext{
${ }^{1}$ Northwestern University, Dept. of Physics \& Astronomy, 2145 Sheridan Rd., Evanston, IL 60208; cheinke@ northwestern.edu

${ }^{2}$ Lindheimer Postdoctoral Fellow
}

both of which indicate the presence of a compact object surface for NS systems and not for black hole systems. These NS systems generally show soft spectra, consisting of a thermal, blackbody-like component, and possibly a harder component extending to higher energies, usually fit with a power-law of photon index 1-2 (although some systems are dominated by the harder component; Campana et al. 2002; Wiinands et al. 2005a). The thermal component, if fit by a blackbody, produces inferred radii too small for theoretical NS size estimates. However, an accreting NS will develop a pure hydrogen atmosphere if the accretion rate falls below $\sim 10^{-13}$ $M_{\odot} \mathrm{yr}^{-1}$ (Brown et al. 1998), because the metals settle out of the atmosphere within a few seconds (Romani 1987). Raiagopal \& Romani (1996) and Zavlin et al. (1996) showed that hydrogen NS atmospheres shift the peak of the emitted radiation to higher frequencies due to the strong frequency dependence of free-free absorption.

A major unsolved question about qLMXBs is the nature of the X-ray emission. Brown et al. (1998) advanced the idea (also discussed by Campana et al. 1998) that the soft thermal component seen in these field systems can be explained by the release, over long timescales, of heat injected into the deep crust by pycnonuclear reactions driven during accretion (the "deep crustal heating" model). This scenario generally predicts the quiescent thermal luminosity of many qLMXBs, based on their outburst history, reasonably well (Rutledge et al. 2001b), but not for all qLMXBs (cf. Colpi et al. 2001; Campana et al. 2002; Wiinands et al. 2005a). The deep crustal heating model cannot explain the hard power-law component, which is often attributed to continued accretion and/or a shock from a pulsar wind (Campana et al. 1998; Bogdanov et al. 2005). The deep crustal heating model also cannot explain the shorttimescale $\left(\sim 10^{4} \mathrm{~s}\right)$ variability observed from Aquila X-1 (Rutledge et al. 2002b) and Cen X-4 (Campana et al. 2004). 
Continued accretion has been suggested as an explanation for the thermal component, as the radiation spectrum from matter accreting radially onto a neutron star should be similar to that expected from deep crustal heating (Zampieri et al. 1995). If an absorption feature due to metals in the NS atmosphere were to be confirmed in a qLMXB spectrum (as suggested in Rutledge et al. 2002b), this would provide evidence for continued accretion at rates sufficient to explain most or all of the thermal emission.

Applying the hydrogen-atmosphere NS models of Zavlin et al. (1996) to Chandra observations of qLMXBs, Rutledge et al. (1999, 2001a b) have shown that the radius predictions of the models are consistent with the range of radii expected from NSs. These analyses have suffered from uncertainties in the distances to qLMXB systems, and from uncertainties due to fitting two components (the power-law plus thermal components) to a spectrum. The distance is tightly constrained for some globular clusters (4\% distance uncertainty to 47 Tuc, Gratton et al. 2003), making them an excellent target for such studies, as pioneered by Rutledge et al. (2002a); Gendre et al. (2003b); Heinke et al. (2003). The well-studied globular cluster 47 Tucanae (NGC 104; hereafter 47 Tuc) is especially ideal due to its close distance $(4.85 \pm 0.18 \mathrm{kpc})$, low reddening $(E(B-V)=0.024 \pm 0.004$, Gratton et al. 2003), presence of two reasonably bright qLMXBs (X5 and X7; Heinke et al. 2003, hereafter HGL03), and deep Chandra observations (300 ksec, Heinke et al. 2005).

To match the quality of the best Chandra data we desire highly accurate hydrogen-atmosphere NS models. We have been troubled by the disagreement between the predictions of the currently available models of Zavlin et al. (1996) and Gänsicke et al. (2002). We also wished to verify whether the variation in surface gravity over the relevant range in NS mass and radius has a significant effect on the atmosphere models and spectral fitting. For these reasons we have produced grids of new hydrogen-atmosphere models. We consider only models of pure hydrogen and for which the magnetic field is sufficiently weak $\left(B \lesssim 10^{8} \mathrm{G}\right)$ that it may be ignored in determining the spectrum. The former assumption is consistent with previous observations of qLMXBs, as iron or solar-abundance atmospheres would be easily identifiable by their different spectral shapes (however, subtle departures from pure $\mathrm{H}$ might still go unnoticed, and affect our results). The latter assumption is consistent with the lack of observable millisecond time variability in qLMXBs similar to X7 (e.g. Aql X-1, Chandler \& Rutledge 2000); the accreting millisecond X-ray pulsars appear to be substantially fainter and harder in quiescence (Wiinands et al. 2005b).

A number of codes exist to compute the spectrum for the simple case of a pure hydrogen NS atmosphere with no magnetic field. Some of these are limited to zero magnetic field, e.g., Raiagopal \& Romani (1996), Gänsicke et al. (2002), and McClintock et al. (2004). Others include magnetic fields, but can be applied in the zero field limit, e.g., Zavlin et al. (1996) and Llovd (2003).

Even with these simplifying assumptions, grids of NS atmosphere models over a wide range of parameters are not widely available for use in XSPEC. Some researchers have computed their own model atmospheres to be used in XSPEC and have included ranges of gravity as well as effective temperature, e.g., Zavlin et al. (1998), using NSA, and Stage et al. (2004), using ATM. However, the available models included in the standard
XSPEC package, namely $\operatorname{NSA}\left(g_{s}\right)$ (Zavlin et al. 1996) and HYD_SPECTRA $\left(g_{s}\right)$ (Gänsicke et al. 2002) cover a range of effective temperatures, but only for the single surface gravity $\log g_{s}=14.385$, corresponding to a "standard" NS model with mass $M_{\mathrm{ns}}=1.4 M_{\odot}$ and radius $R_{\mathrm{ns}}=10 \mathrm{~km}$ [we shall emphasize this using the qualifier " $\left(g_{s}\right)$ "]. However, as the results of this paper will demonstrate, the surface gravity can play an important role in the spectral fitting and needs to be taken into account.

A large part of the present effort was devoted to the construction of grids of models to fulfill this need. The need for NS models covering an extensive set of parameters, including surface gravity, motivated us to adapt the code NSATMOS. This code was previously used by McClintock et al. (2004) to investigate the hydrogen atmospheres of hypothetical, compact objects, some so compact that they lay within their own photon spheres; this required taking account of the self-irradiation of the surface due to gravitational bending of rays. The same model assumptions described in the Appendix of McClintock et al. (2004) apply here, with some improvements: the code now includes the radiation force in the equation of hydrostatic equilibrium (e.g. Pavlov et al. 1991), and includes heat conduction by electrons in the energy equation (e.g. Rajagopal \& Romani 1996). These and other technical improvements made to NSATMOS are discussed in Appendix A.

As it turned out for the present problem, neither electron conduction nor radiation force played any substantial role, in agreement with the results of, e.g., Gänsicke et al. (2002). Also, the range of parameters where self-irradiation occurs does not overlap with the range allowing neutron stars which obey causality, indicating self-irradiation is unlikely to be relevant for neutron stars. On the other hand, allowing for variations in surface gravity turned out to be very important. A major advantage of NSATMOS for this problem was its speed, which allowed us to compute extensive grids of models to cover the ranges of effective temperature and gravity tailored to our needs. Our NSATMOS code will be made available to the astronomical community through the XSPEC website ${ }^{3}$.

Our Chandra observations are described in $\$ 2$ We compare the models and data in $\$ 3$, and discuss the implications in $\$ 4$ Our new neutron star atmosphere models are described in detail in Appendix A, and the effects of our consideration of varying surface gravity are discussed in Appendix B.

\section{OBSERVATIONS}

The data used in this paper are from the 2000 and 2002 Chandra observations of the globular cluster 47 Tuc. Both sets of observations and their initial reduction are described in detail in Heinke et al. (2005); prior analyses of the 2000 dataset are described in Grindlay et al. (2001) and HGL03. The 2000 observations were performed with the ACIS-I CCD array at the telescope focus, while the 2002 observations placed the back-illuminated ACIS-S aimpoint at the focus for maximum low-energy sensitivity. Five consecutive observations were performed in 2000, as listed in Table 1, with three short observations, using a subarray and faster readout time, interleaved to reduce pileup in bright sources such as X7 and X5 (see HGL03). Pileup occurs when two X-ray photons, arriving at the detector during one frame time, are erroneously identified as a single photon with the sum of the two photon energies, or else discarded (see Davis 2001). Pileup

\footnotetext{
${ }^{3}$ http://heasarc.gsfc.nasa.gov/docs/xanadu/xspec/
} 
TABLE 1

SUMMARY OF Chandra OBSERVATIONS

\begin{tabular}{lcccrr}
\hline \hline Seq, OBSID & Start Time & Exposure & Aimpoint & Frametime & CCDs \\
\hline 300003,078 & 2000 Mar 16 07:18:30 & 3875 & ACIS-I & 0.94 & $1 / 4$ \\
300028,953 & 2000 Mar 16 08:39:44 & 31421 & ACIS-I & 3.24 & 6 \\
300029,954 & 2000 Mar 16 18:03:03 & 845 & ACIS-I & 0.54 & $1 / 8$ \\
300030,955 & 2000 Mar 16 18:33:03 & 31354 & ACIS-I & 3.24 & 6 \\
300031,956 & 2000 Mar 17 03:56:23 & 4656 & ACIS-I & 0.94 & $1 / 4$ \\
400215,2735 & 2002 Sep 29 16:59:00 & 65237 & ACIS-S & 3.14 & 5 \\
400215,3384 & 2002 Sep 30 11:38:22 & 5307 & ACIS-S & 0.84 & $1 / 4$ \\
400216,2736 & 2002 Sep 30 13:25:32 & 65243 & ACIS-S & 3.14 & 5 \\
400216,3385 & 2002 Oct 01 08:13:32 & 5307 & ACIS-S & 0.84 & $1 / 4$ \\
400217,2737 & 2002 Oct 02 18:51:10 & 65243 & ACIS-S & 3.14 & 5 \\
400217,3386 & 2002 Oct 03 13:38:21 & 5545 & ACIS-S & 0.84 & $1 / 4$ \\
400218,2738 & 2002 Oct 11 01:42:59 & 68771 & ACIS-S & 3.14 & 5 \\
400218,3387 & 2002 Oct 11 21:23:12 & 5735 & ACIS-S & 0.84 & $1 / 4$ \\
& & & & &
\end{tabular}

NOTE. - Times in seconds. Subarrays are indicated by fractional numbers of CCDs. We do not use OBS_ID 3385 in this paper, due to its relatively high background.

has effects upon both spectral and timing analyses, as discussed in the Chandra Proposers' Observatory Guide ${ }^{4}$ and Davis (2001).

The 2002 observations also interleaved long observations with shorter ones to collect some data relatively free of pileup. The countrates for X7 in the 2000 and 2002 observations (both essentially on-axis) were 0.07 and $0.12 \mathrm{cts} / \mathrm{s}$, leading to predicted pileup rates of $9 \%$ and $15 \%$ for full-array observations, or $2 \%$ and $4 \%$ using $1 / 4$ subarrays. We reprocessed the 2000 observations using the CTI correction algorithm implemented in CIAO 3.2 acis_process_events. Both the 2000 and 2002 observations were reprocessed (using CIAO 3.2) to remove the $0 . " 5$ pixel randomization added in standard processing, use updated (time-dependent) gain files, and improve hot pixel identifications. Some background flaring occurred during parts of the 2002 observations, particularly affecting OBS_ID 3385. We do not use data from that short $(5 \mathrm{ksec})$ observation, but keep all other data.

We used the ACIS_EXTRACT software (Broos et al. 2002), version 3.65, to extract and combine spectra and response files for the various observations. We extracted spectra from contours matching the $95 \%$ encircled energy (at 1.5 $\mathrm{keV}$ ) of the Chandra point-spread function at X7's position. We constructed response files using the mkacisrmf response generator in CIAO 3.2. The effective area files take into account the decreasing quantum efficiency of the ACIS chips, and are corrected to account for the energy-dependent fraction of the point-spread function enclosed by the extraction region (Broos et al. 2002). We combined spectra from: the two long 2000 observations, OBS_IDs 953 and 955; the four long 2002 observations; the three remaining short 2002 observations (see above); and the three short 2000 observations, for a total of four summed spectra. We binned these spectra at 80 counts/bin for the long 2002 spectrum, 20 counts/bin for the short 2000 spectrum, and 40 counts/bin for the other two spectra. 


\subsection{Timing Analysis}

We adjusted all event times to the solar system barycenter using satellite orbit files provided by the Chandra X-ray Center. The qLMXB X5 continues to show eclipses and very strong dipping activity throughout the 2002 observations, which make a study of its spectrum more complicated; we defer detailed studies of X5 to a later paper.

Kolmogorov-Smirnov and Cramer-von Mises tests showed no variability from X7 on any timescales probed by the 2002 observations (seconds to weeks). Power spectra (constructed using XRONOS) of X7's lightcurves showed a flat power spectrum with less power than expected from Poisson processes (Leahy et al. 1983), typically $\sim 70 \%$ of the expected level. We attribute this to the effects of moderate pileup (as other, weaker, X-ray sources in 47 Tuc showed the expected levels of white noise), and note that it makes a quantitative limit on X7's variability difficult to determine. Assuming constant Poisson noise (the constant level was a free parameter), plus red noise with a fixed slope $\left(\propto \nu^{-1}\right.$, e.g. Rutledge et al. 2001a), we constrain $\left(3 \sigma\right.$, between $10^{-5}$ and $\left.0.1 \mathrm{~Hz}\right)$ the rms variability to $<3.6 \%,<7.3 \%,<1.9 \%$ and $<5.2 \%$ for the four 2002 observations. These values may be underestimates due to the effects of pileup. We also extract power spectra from 1-2.' annuli, to reduce the effects of pileup, giving 511 to 541 counts per dataset. These power spectra display white noise at the levels expected from Poisson noise. We again find no evidence for excess variability, with $3 \sigma$ upper limits on rms variability being $<17 \%,<15 \%,<25 \%$ and $<28 \%$ for each observation. The data taken in subarray mode to reduce pileup showed similar properties, with $3 \sigma$ upper limits of $<13 \%,<17 \%$, and $<24 \%$ rms variability (excluding OBS_ID 3385). No signal was seen at 5.50 hours, the period of a marginal signal identified in HGL03, indicating that the suggested orbital period (from the lower-quality 2000 data) is probably spurious.

\section{SPECTRAL ANALYSIS}

\subsection{NSATMOS Spectral Analysis of X7}

Our standard XSPEC model consists of the NSATMOS hydrogen atmosphere model, absorbed by interstellar gas, convolved with the XSPEC pileup model. We use the XSPEC (Arnaud 1996) version of the pileup model of J. Davis (Davis 2001), setting the frame time parameter to the time resolution of each spectrum (header keyword TIMEDEL), and the parameters $g_{0}=1$ and $p s f f r a c=0.95$. We floated the grade migration parameter $\alpha$ (which parametrizes the fraction of piled photons that are recorded as good grades), but found that its value is always consistent with 0.5 , and fixed it to 0.5 for this section and $\$ 3.2$

We use the XSPEC phabs model, with Wilms et al. (2000) interstellar element abundances, to describe the interstellar gas between the Earth and 47 Tuc. We fix its normalization at $N_{H}=1.3 \times 10^{20} \mathrm{~cm}^{-2}$, derived using $\mathrm{E}(\mathrm{B}$ $\mathrm{V})=0.024 \pm 0.004$ as measured by Gratton et al. (2003), and assuming $R_{V}=3.1$ (Cardelli et al. 1989) and $N_{H} / A_{V}=1.79 \times$ $10^{21} \mathrm{~cm}^{-2}$ (Predehl \& Schmitt 1995). The $N_{H}$ column measured using any of our models is larger than this, indicating additional gas intrinsic to the system (or slight errors in the Chandra ACIS calibration below $1 \mathrm{keV}$ ). We model this additional absorbing gas by the XSPEC vphabs model, selecting the abundances to correspond to those of 47 Tuc. We choose the abundance of iron to be $20 \%$ of solar $([\mathrm{Fe} / \mathrm{H}]=-$ 0.7 , that of metals between $\mathrm{Ne}$ and $\mathrm{Ca}$ to be $40 \%$ solar

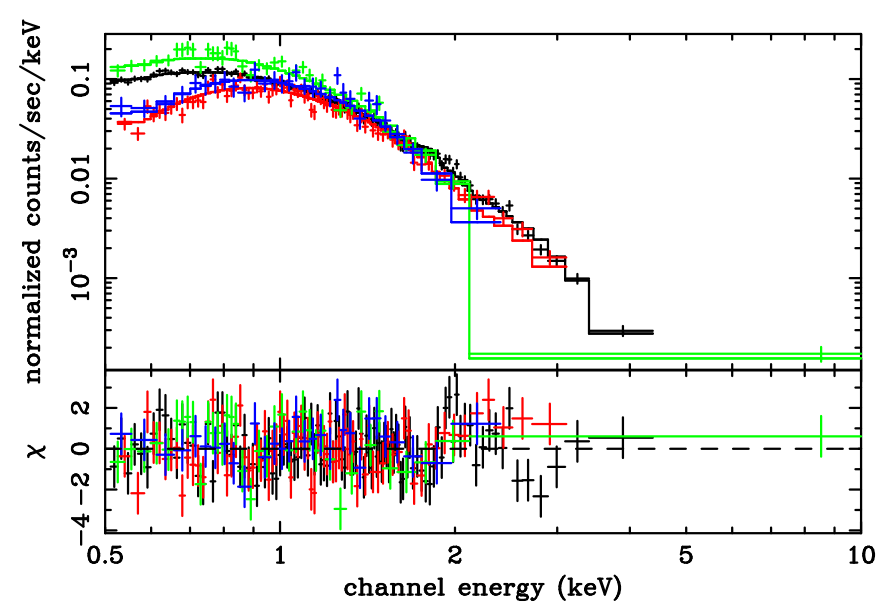

FIG. 1.- From top, Chandra ACIS-S subarray spectrum (green), ACIS-S full-frame spectrum (black), ACIS-I subarray spectrum (blue), and ACIS-I full-frame spectrum (red) of X7, fit with our hydrogen-atmosphere NSATMOS model (histograms) and photoelectric absorption (see text). Pileup is responsible for the variation in count rate between the subarray and full-frame spectra,and is accounted for in the fit. The apparent difference between the full-frame spectra is due to the differing spectral responses of the ACIS-S and ACIS-I instruments, as no model parameter is allowed to vary between the fits. The strongest residuals (near $2 \mathrm{keV}$ ) are instrumental features due to the iridium edges of the mirror and resultant rapid changes in effective area with energy. See the electronic edition of the Journal for a color version of this figure.

$([\mathrm{X} / \mathrm{H}]=-0.4), \mathrm{C}, \mathrm{N}$, and $\mathrm{O}$ to be $63 \%$ solar $([\mathrm{X} / \mathrm{H}]=-0.2)$, and $\mathrm{He}$ at solar abundance (using Carnev 1996; Salaris \& Weiss 1998; Gratton et al. 2003). We note that our results are not very sensitive to the detailed abundances, since the absorption column is low (see below).

The Chandra ACIS effective area and response matrix calibrations are very uncertain below $0.5 \mathrm{keV}$. We find substantial negative residuals around $0.4 \mathrm{keV}$, similar to an absorption line (but substantially narrower than the instrument resolution). This feature is seen in most other X-ray sources of sufficient flux in 47 Tuc (Heinke et al. 2005), so we ascribe it to an instrumental effect and ignore data below $0.5 \mathrm{keV}$ in this paper. A second feature is a wave in the residuals between 1.7 and $2.3 \mathrm{keV}$. This feature can be ascribed to the difficulty of calibrating the iridium M edge structure (Chandra Proposer's Observatory Guide), and similar waves are seen in other highquality ACIS CCD spectra (Sanders et al. 2004). This residual causes the quality of our best fits to be slightly less than nominal, $\chi_{\nu}^{2}=1.20$ for a null hypothesis probability (nhp) of $1.5 \%$. More acceptable fits $(\mathrm{nhp}=7 \%)$ can be achieved by adding a systematic error term (of order $2 \%$ ), or by excluding the spectral range from 1.9-2.25 keV in the two highestquality spectra, but the parameters of the fit do not change substantially from the fits using all the data.

Fitting the four combined X7 spectra simultaneously in XSPEC, leaving no parameters free between the various datasets, and only the intrinsic absorption, NS temperature, and NS radius as free parameters, gives a reasonably good fit $\left(\chi_{\nu}^{2}=1.20, \mathrm{nhp}=1.5 \%\right.$; see Fig. 11. (To begin with, we fix the NS mass at $1.4 M_{\odot}$. We will vary this later.) The inferred (unabsorbed) luminosity of X7 is $L_{X}(0.5-10 \mathrm{keV})=1.5 \times 10^{33}$ $\operatorname{ergs~s}{ }^{-1}, L_{\mathrm{bol}}=2.4 \times 10^{33} \mathrm{ergs} \mathrm{s}^{-1}$. Allowing the absorption column or the NS temperature, radius, normalization, or distance to vary between the 2002 and 2000 observations does not improve the fit, and the best-fit values for each obser- 
vation lie within the one-sigma errors of the best fit for the other observation. A quantitative constraint on X7's spectral variability between the 2000 and 2002 observations can be obtained by decoupling the 2000 and 2002 temperatures or normalizations, and measuring how large the difference may be; we find $k T_{X 7,2000}=1.003_{-0.006}^{+0.006} \times k T_{X 7,2002}$, or for normalization, $K_{X 7,2000}=1.01_{-0.03}^{+0.03} \times K_{X 7,2002}(90 \%$ conf.). Fitting the individual spectra extracted for each of the four long 2002 observations (grouped at 20 counts/bin) with the same model gives a good fit $\left(\chi_{\nu}^{2}=0.962, \mathrm{nhp}=70 \%\right)$, and when the model parameters are allowed to vary between observations they again agree with one another. (The improvement in the fit quality, compared to the combined spectrum, is due to the reduced statistics of each observation individually.) Therefore we conclude that these deep observations provide no evidence for any spectral change in X7 over a period of 2.5 years. This result is in contrast to other qLMXBs which have displayed substantial variability in quiescence (Rutledge et al. 2002b; Campana et al. 2004).

\subsection{Edge or power-law?}

HGL03 found marginal evidence for the existence of an edge or other absorption feature near 0.63 and $0.66 \mathrm{keV}$ in $\mathrm{X} 7$ and X5, respectively. No such feature is apparent in the 2002 X7 data, and the evidence for such a feature from the 2000 data has decreased as the Chandra calibration has improved. Fitting all the X7 data with the standard model above and an edge fixed at $0.63 \mathrm{keV}$ (as in HGL03) does not improve the fit, and leads to a $90 \%$ confidence upper limit of $\tau<0.045$ for the edge. The two explanations for the edge suggested by HGL03 (an OV edge from an ionized wind, or a signature of a NS atmosphere that is not purely hydrogen) can both be excluded by the disappearance of this feature in the 2002 dataset. We feel that the most likely explanation for this apparent feature is small uncertainties in the calibration.

The existence of any spectral feature in a qLMXB spectrum would be of great interest, since an identification of the feature could allow determination of the gravitational redshift at the NS surface (Brown et al. 1998; Rutledge et al. 2002b HGL03). Therefore we searched for any possible features in X7's spectrum, using an edge or a gaussian absorption line, between 0.55 and $3 \mathrm{keV}$. The largest possible features were at $0.85 \mathrm{keV}$, where an edge with $\tau=0.065_{-0.05}^{+0.07}$ gave $\chi_{\nu}^{2}=1.017 ; 1.58 \mathrm{keV}$, where an edge with $\tau=0.14_{-0.10}^{+0.11}$ gave $\chi_{\nu}^{2}=1.194 ;$ and $2.54 \mathrm{keV}$, where an edge with $\tau=0.70_{-0.7}^{+1.9}$ gave $\chi_{\nu}^{2}=1.204$. The latter two are probably caused by the calibration uncertainties around $2 \mathrm{keV}$. These features have F-test probabilities (to be interpreted with caution) of $7.7 \%$, $13 \%$, and $45 \%$ of being generated by chance, which are consistent with the low significances of their optical depths (none nonzero at more than $98 \%$ confidence). The $90 \%$ confidence limits on edge equivalent widths at these locations are $<18$, $<29$, and $<53 \mathrm{eV}$. Using a gaussian absorption line with fixed intrinsic width of $0.05 \mathrm{keV}$, we find similar results, with the most likely features located at $0.92\left(\tau=0.083_{-0.05}^{+0.05}\right)$ or 1.73 $\left(\tau=0.33_{-0.15}^{+0.20}\right) \mathrm{keV}$. Equivalent width upper limits are $<11$ and $<33 \mathrm{eV}$, respectively.

The feature at $\sim 0.4 \mathrm{keV}$ discussed above is the strongest apparent feature. The anticipated energy of the strongest feature (due principally to the O VIII edge) likely to appear on an accreting neutron star with a near-solar abundance atmosphere is $0.87 /(1+z) \mathrm{keV}$ (Brown et al. 1998; Rutledge et al. 2002b see their Fig. 1). There are no plausible features expected near
$0.4 \mathrm{keV}$ for gravitational redshifts in the range 0.2-0.4 (typical for favored neutron star equations of state). We conclude that no spectral features intrinsic to the NS atmosphere have been detected.

The hard power-law component identified in many field qLMXBs may be a signal of continued accretion (Rutledge et al. 2002b), or of nonthermal emission from the pulsar wind of an underlying MSP (Burderi et al. 2003; Bogdanov et al. 2005). We constrain such a power-law component by adding it to our standard model with a fixed photon index $\Gamma$ of $1.5,2$ or 1 , and deriving constraints upon its flux in the $0.5-10 \mathrm{keV}$ band relative to the total (absorbed) $0.5-10$ $\mathrm{keV}$ flux. Adding this component does not significantly improve the fit $\left(\chi_{\nu}^{2}=1.205\right.$, an F-test suggests a $60 \%$ chance of this level of improvement by chance). For $\Gamma=1.5$, such a power-law component would make up $<3.2 \%$ (90\% conf.) of X7's total $0.5-10 \mathrm{keV}$ flux. For $\Gamma=2$ or 1 , the constraints are $<3.4 \%$ of the total $0.5-10 \mathrm{keV}$ flux.

The complete lack of evidence for variability, edges, or hard power-law components in X7 suggests that X7's X-ray emission is produced entirely through re-emission of stored heat (Brown et al. 1998). The constraints on these properties for $\mathrm{X} 7$, more stringent than for any other qLMXB, make this an ideal object for comparisons with NS hydrogen-atmosphere models.

\subsection{Constraining the NS Mass and Radius}

The principal goal of our spectral fitting is to selfconsistently constrain the allowed space in mass and radius of X7's neutron star. First we explore the best fits for mass and radius if one parameter is fixed at a canonical value, $M_{\mathrm{ns}}=1.4 M_{\odot}$ or $R_{\mathrm{ns}}=10 \mathrm{~km}$. Our standard model above keeps the mass fixed at $1.4 M_{\odot}$, and the fitted radius is $14.2_{-1.0}^{+1.1}$ $\mathrm{km}(90 \%$ conf.). Allowing the pileup parameter $\alpha$ to vary, and allowing for a power-law component with photon index fixed at 1.5 , the fitted radius is $14.5_{-1.4}^{+1.6} \mathrm{~km}$. The parameters for this fit are included in Table 2 . We note that a pure helium atmosphere (possible if the donor is degenerate) would give even larger radii (Zavlin et al. 1996; Pons et al. 2002), which we think unlikely. Adding a $6.1 \%$ uncertainty (Gratton et al. 2003, 90\% conf., assuming errors are normally distributed) in the distance to 47 Tuc gives a NS radius of $14.5_{-1.6}^{+1.8} \mathrm{~km}$. The lower limit to X7's radius is thus 12.9 $\mathrm{km}$, which is significantly larger than the radius predicted by the APR (11.5 km, Akmal et al. 1998) and FPS (10.8 km, Pandharipande \& Ravenhall 1989) equations of state for a 1.4 $M_{\odot}$ NS.

If we instead allow $M_{\mathrm{ns}}$ to vary and fix $R_{\mathrm{ns}}$ at $10 \mathrm{~km}$, we find that $M_{\mathrm{ns}}$ is constrained to $2.17_{-0.12}^{+0.10} M_{\odot}$; allowing $\alpha$ to vary and including a possible powerlaw component, $M_{\mathrm{ns}}=2.20_{-0.15}^{+0.16} M_{\odot}$. Adding distance uncertainty, the errors are $M_{\mathrm{ns}}=2.20_{-0.16}^{+0.18} M_{\odot}$. Constraining the neutron star mass to lie below the causality line gives $M_{\mathrm{ns}}=2.20_{-0.16}^{+0.03} M_{\odot}$. This mass is significantly larger than that of any well-measured NS (but cf. Nice et al. 2005). A high mass for X7 seems improbable given its relatively high X-ray luminosity (compared to other qLMXBs), and the expected tendency for more massive NSs to cool more quickly (Yakovlev \& Pethick 2004). We give the parameters for fits with either $M_{\mathrm{ns}}$ or $R_{\mathrm{ns}}$ held fixed in Table 2, including all uncertainties except for the $6 \%$ distance uncertainty.

We use the steppar command in XSPEC to vary both the radius and mass parameters, allowing all other free parameters (including $\alpha$ and the powerlaw normalization) to 
TABLE 2

X7 Spectral Model Parameters

\begin{tabular}{|c|c|c|}
\hline Model Parameter & M fixed & $\mathbf{R}$ fixed \\
\hline \multicolumn{3}{|c|}{ Rybicki NSATMOS model } \\
\hline$k T, \mathrm{eV}$ & $105.4_{-56}^{+5.6}$ & $151.5_{-59}^{+7.4}$ \\
\hline$N_{H}^{a}$ & $4.2_{-16}^{+1.5 .6}$ & $4.0_{-15}^{+1.8 .9}$ \\
\hline$R_{\mathrm{ns}}, \mathrm{km}$ & $14.5_{-1.4}^{+1.6}$ & $(10.0)$ \\
\hline$M_{\mathrm{ns}}$ & $(1.4)^{-1.4}$ & $2.20_{-0.15}^{+0.16}$ \\
\hline$\chi_{u}^{2} / \mathrm{dof}$ & $1.21 / 251$ & $1.21 / 251$ \\
\hline Null hyp. prob. & $1.3 \%$ & $1.3 \%$ \\
\hline \multicolumn{3}{|c|}{ Zavlin NSA $\left(g_{s}\right)$ model } \\
\hline$k T, \mathrm{eV}$ & $89^{+5}$ & $145_{5}^{+4}$ \\
\hline$N_{H, 20}^{a}$ & $5.6_{-0.4}^{+0.8}$ & $3.5_{-2.8}^{+5.0}$ \\
\hline$R_{\mathrm{ns}}, \mathrm{km}$ & $19.9_{-2.1}^{+0.1^{b}}$ & $(10.0)$ \\
\hline$M_{\mathrm{ns}}$ & $(1.4)$ & $1.99_{-0.15}^{+0.13}$ \\
\hline$\chi_{\nu}^{2} / \mathrm{dof}$ & $1.21 / 251$ & $1.21 / 251$ \\
\hline Null hyp. prob. & $1.2 \%$ & $1.3 \%$ \\
\hline \multicolumn{3}{|c|}{ Gänsicke HYD_SPECTRA $\left(g_{s}\right)$ model } \\
\hline- & $\mathrm{Z}=0.306$ & R fixed \\
\hline$k T, \mathrm{eV}$ & $123^{+4}$ & $138^{+5}$ \\
\hline$N_{H, 20}^{a}$ & $5.1_{-0.9}^{+0.5}$ & $4.4_{-0.8}^{+-4.6}$ \\
\hline$R_{\mathrm{ns}}, \mathrm{km}$ & $11.9_{-1.2}^{+1.9}$ & $(10.0)$ \\
\hline$M_{\mathrm{ns}}$ & $1.66_{-0.15}^{+0.22}$ & $1.68_{-0.17}^{+0.20}$ \\
\hline$\chi_{\nu}^{2} / \mathrm{dof}$ & $1.21 / 251$ & $1.21 / 251$ \\
\hline Null hyp. prob. & $1.3 \%$ & $1.3 \%$ \\
\hline
\end{tabular}

NOTE. - All errors are $90 \%$ confidence limits. Distance of $4.85 \mathrm{kpc}$ is assumed (its uncertainty is not included in these quoted errors). In each column, either mass, redshift $(z)$, or the true radius of the neutron star is held fixed. ${ }^{a} N_{H}$ intrinsic to system, in units of $10^{20} \mathrm{~cm}^{-2}$, in addition to galactic column of $1.3 \times 10^{20} \mathrm{~cm}^{-2} \cdot{ }^{b}$ Reached hard limit of model.

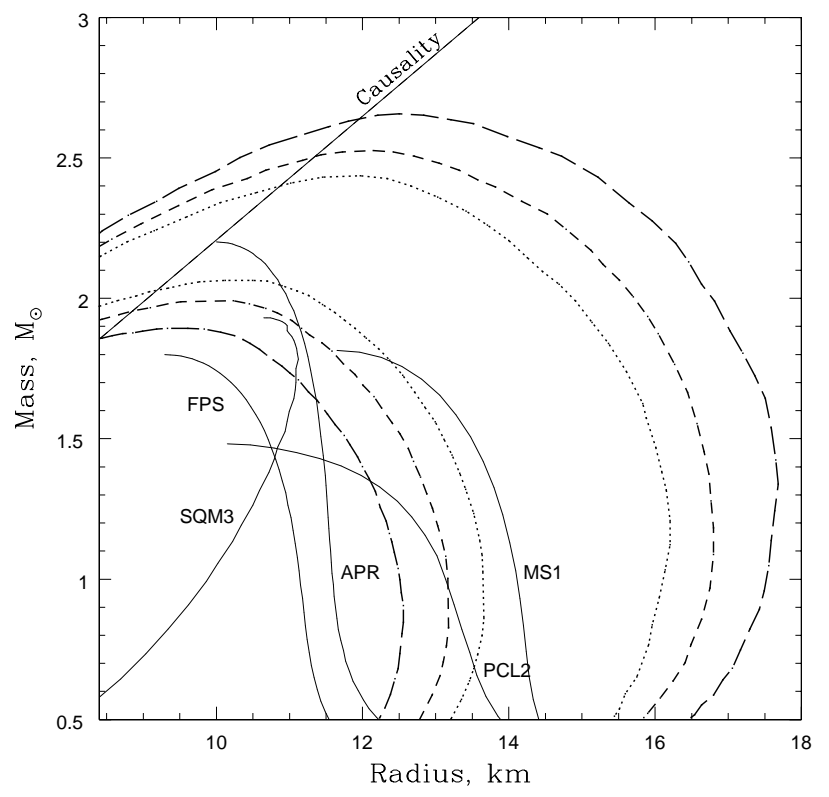

FIG. 2.- 68\% (dotted), 90\% (short dash) and 99\% (long dash) confidence contours in the mass-radius plane derived for $\mathrm{X} 7$ by our spectral fitting with the NSATMOS model. The causality line, above which no realistic NS equations of state can exist, is plotted, along with five representative equations of state, APR, FPS, MS1, PCL2, and SQM3 (the last a quark star model, see Lattimer \& Prakash 2001). vary to find the best fit. We show $1 \sigma, 90 \%$ confidence, and $99 \%$ confidence $\left(\Delta \chi^{2}=2.3,4.61\right.$, and 9.21 respectively) contours in NS mass and radius in Fig. 2 for X7, using our spectral model as described above. These can be compared with the loci of models following the APR (Akmal et al. 1998), FPS (Pandharipande \& Ravenhall 1989), PCL2 (Prakash et al. 1995), MS1 (Müller \& Serot 1996), and SQM3 (Prakash et al. 1995) equations of state for dense matter (the last is a representative "quark star" model). For the SQM3 and APR models, a high NS mass is required for consistency with the data; the FPS model is ruled out; and the PCL2 model is marginally consistent with the data at the $99 \%$ level for a mass of $1.35 M_{\odot}$. The MS1 model is an example of models consistent with a larger radius at $1.4 M_{\odot}$ (see Lattimer \& Prakash 2001), some of which include condensation of kaons, hyperons, muons or free quarks in the core. The line labeled "Causality" $\left(R=3.04 G M / c^{2}\right)$ represents the requirement that the speed of sound must be less than $c$, a necessary (but not sufficient) requirement for a NS interior to respect causality (Lattimer \& Prakash 2001; Olson 2001). This requirement is virtually identical to $R>3 G M / c^{2}$, which is the condition that the neutron star surface is outside its own photon sphere, so that self-irradiation does not occur.

\subsection{Comparison of NS atmosphere models}

We compare three different hydrogen-atmosphere neutron star models in this analysis. Our NSATMOS model has parameters $M_{\mathrm{ns}}$ (mass of neutron star, $M_{\odot}$ ), $R_{\mathrm{ns}}$ (true radius of the neutron star, $\mathrm{km}$ ), $T_{\text {eff }}$ (the effective temperature of the neutron star surface, unredshifted), and $D$ (distance to neutron star, pc). The NSA model (Zavlin et al. 1996) has parameters $R$ (true radius of the neutron star, $\mathrm{km}$ ), $M$ (mass of neutron star, $\left.M_{\odot}\right), T_{\text {eff }}$ (the effective temperature of the neutron star surface, unredshifted), and normalization $K=1 / D^{2}$ (where $D$ is the distance to the neutron star, pc). The last parameter is often used to calculate $R_{\infty}=R(1+z)$, the radius as seen by a distant observer, since the last parameter can be considered $K=\left(R_{\infty} / R_{\infty, 0}\right)^{2} / D^{2}$, where $R_{\infty, 0}$ is the equivalent $R_{\infty}$ that would be calculated from the model parameters $R$ and $M$. The HYD_SPECTRA model of Gänsicke et al. (2002) has the parameters $T_{\text {eff }}, z$ (the gravitational redshift at the NS surface), and normalization $K=(R / D)^{2}$, where $R$ is in units of $10 \mathrm{~km}$ and $D$ is measured in pc. We also tested the H_ATM model of Llovd (2003), for which the parameters are $T_{\text {eff }}, z$, and normalization $K=(R / D)^{2}$, where $R$ is in $\mathrm{km}$ and $D$ is in units of $10 \mathrm{kpc}$. The H_ATM model gives results broadly similar to those of the NSA model, and for brevity we will not discuss H_ATM further in this paper. The NSATMOS and NSA models fit directly for $M_{\mathrm{ns}}$ and $R_{\mathrm{NS}}$, while the HYD_SPECTRA model fits redshift and normalization, which allows computation of $M_{\mathrm{ns}}$ and $R_{\mathrm{ns}}$.

While the NSA and HYD_SPECTRA codes can, in principle, be used to compute models for any value of gravity, the actual versions of these models found in the standard XSPEC package have been constructed for single values of the surface gravity $g_{s}$ (appropriate for a $1.4 M_{\odot}, 10 \mathrm{~km} \mathrm{NS}$ ). To emphasize this, we shall denote these models by $\operatorname{NSA}\left(g_{s}\right)$ and HYD_SPECTRA $\left(g_{s}\right)$. The use of these fixed gravity models for other values of surface gravity is not strictly appropriate. In order to account for the effect of surface gravity on the spectra, we computed an extensive grid of models using the NSATMOS code described in Appendix A. This grid was used as the basis of an interpolation routine that produced model spectra for the XSPEC program for a full range of ef- 


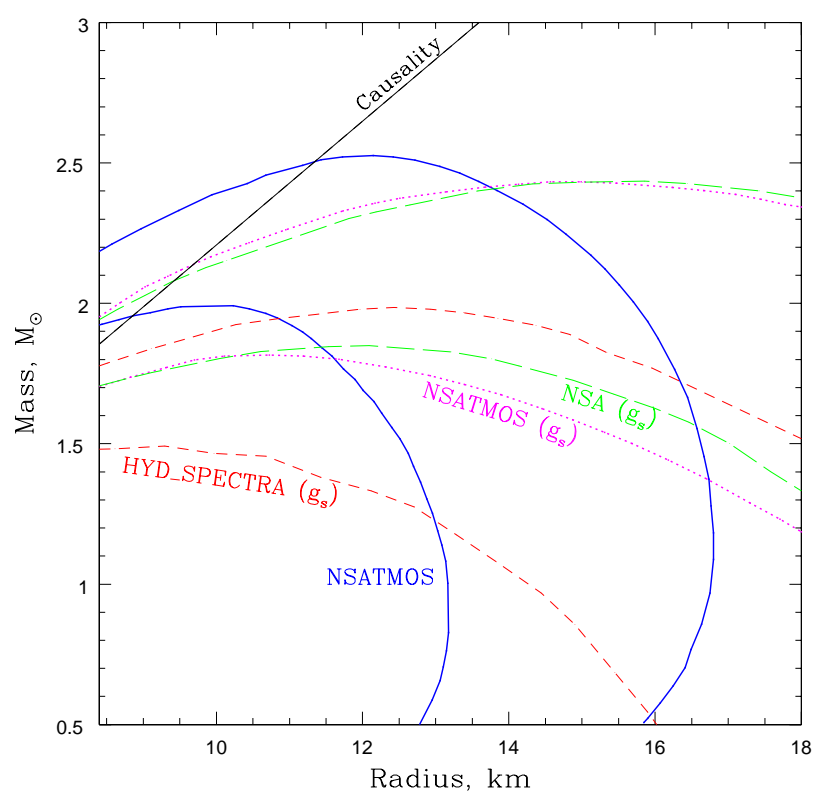

FIG. 3. - 90\% confidence contours in the mass-radius plane derived for X7 by our spectral fitting with hydrogen atmosphere NS models: NSATMOS, using variable $g$, with thick solid (blue) lines, NSA $\left(g_{s}\right)$ (Zavlin et al. 1996) with dashed (green) lines, HYD_SPECTRA $\left(g_{s}\right)$ (Gänsicke et al. 2002) with shortdashed (red) lines, and, for comparison, NSATMOS $\left(g_{s}\right)$, with thick dotted (magenta) lines. Here $g_{s}=14.385$ is the surface gravity for the standard NS model. See the electronic edition of the Journal for a color version of this figure.

fective temperatures and gravities.

We fit each of these three models to our X7 data using the same spectral model as described in section $\$ 3.3$ replacing only the neutron-star atmosphere model. For constraints upon the NS mass, radius, or gravitational redshift, we place the parameters of these fits in Table 2. We plot the contours enclosing $90 \%$ confidence contours for various models together in Fig. 3 The contours labelled NSATMOS were constructed using the appropriate surface gravity for each point in the plot. We have also plotted the contours assuming a fixed standard gravity $\log g_{s}=14.385$ for three codes: $\operatorname{NSATMOS}\left(g_{s}\right)$, $\operatorname{NSA}\left(g_{s}\right)$, and HYD_SPECTRA $\left(g_{s}\right)$, the latter two using the models in the XSPEC package.

We see that none of the available models allow a $1.4 M_{\odot}, 10$ $\mathrm{km}$ radius NS, requiring a more massive or larger radius NS. [However, the small additional uncertainty on the distance to 47 Tuc-not included in Fig. 3-allows marginal consistency at the $99 \%$ level with the HYD_SPECTRA model $\left(g_{s}\right)$.] Since the HYD_SPECTRA $\left(g_{s}\right)$ and $\operatorname{NSA}\left(g_{s}\right)$ models are constructed with a $g_{s}$ appropriate for a $1.4 M_{\odot}, 10 \mathrm{~km} \mathrm{NS}$, the failure of those models to find an acceptable fit for a 1.4 $M_{\odot}, 10 \mathrm{~km} \mathrm{NS}$ is robust. It is not clear to us why the HYD_SPECTRA $\left(g_{s}\right)$ model predictions are significantly different from the other two models, although the difference between the HYD_SPECTRA and NSA hydrogen models has been previously noted (Gänsicke et al. 2002). Caution should still be taken in interpreting our results due to possible unknown systematic uncertainties in the à̀Chandra calibration. However, the robustness of all tested models in excluding the canonical mass and radius values $\left(1.4 M_{\odot}\right.$ and $\left.10 \mathrm{~km}\right)$ is strong evidence for a relatively "stiff" NS equation of state.

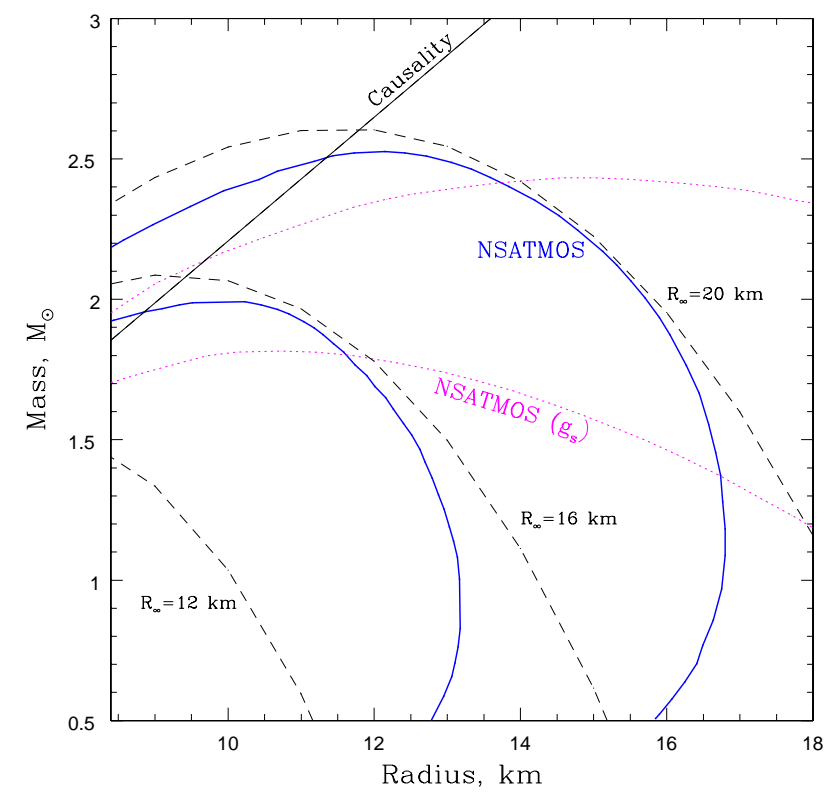

FIG. 4.- 90\% confidence contours in the mass-radius plane derived for X7 by our spectral fitting with two versions of our hydrogen atmosphere NS models: NSATMOS (variable $g$ ) and NSATMOS $\left(g_{s}\right)$, compared to lines of constant $R_{\infty}$. See the electronic edition of the Journal for a color version of this figure.

\section{DISCUSSION}

In Fig. 4 we compare the allowed contours of mass and radius for $\mathrm{X} 7$ using the full NSATMOS and fixed-gravity NSATMOS $\left(g_{s}\right)$ models to lines of constant $R_{\infty}$ for a blackbody spectrum (or for any spectrum of the scaled form in equation [B1]). The contours derived from our grid of NSATMOS models lie closer to lines of constant $R_{\infty}$ than do the contours derived from the fixed-gravity models, but there are significant differences. (See Appendix B for further discussion of the surface gravity effects.)

The effect of self-consistently including variation in $g_{s}$ is quite substantial, as can be seen from the difference between our NSATMOS contours and the contours of NSATMOS $\left(g_{s}\right)$. The predictions of the $\operatorname{NSA}\left(g_{s}\right)$ model are very similar to those of our NSATMOS $\left(g_{s}\right)$ model, demonstrating that variation in surface gravity is the primary reason for the differences in the results obtained with NSATMOS and NSA. This demonstrates that the NSA $\left(g_{s}\right)$ and HYD_SPECTRA $\left(g_{s}\right)$ models currently available in XSPEC do not give correct constraints in regions of parameter space where the assumed surface gravities are different than those for which they were computed, and which are outside their region of validity. Explicitly, the constraints on neutron star mass and radius computed by HGL03 used these models outside their range of validity, and produced incorrect results. It is possible that this error in assumptions may affect the results of Pons et al. (2002) and Walter \& Lattimer (2002) as well.

Some works have used the NSA models in XSPEC to constrain the range of $R_{\infty}$ (Rutledge et al. 2001b a; Gendre et al. $2003 \mathrm{~b}$ a). This method is more accurate due to a degeneracy in spectral shape variations between surface gravity and surface temperature (see Zavlin et al. 1998, and Appendix B). For instance, fitting X7 with the NSA model, freezing the mass $\left(1.4 M_{\odot}\right)$ and radius $(10 \mathrm{~km})$, we infer $R_{\infty}=18.34$ 
$\mathrm{km}$, with a $90 \%$ confidence range between 17.1 and $22.1 \mathrm{~km}$. Comparing this to Figure 4, we see that this range is more accurate than the method of HGL03, though still inaccurate at the $\sim 10 \%$ level. The differences between hydrogen atmosphere spectra and blackbodies are large enough that direct calculation of a range of $M_{\mathrm{ns}}$ and $R_{\mathrm{ns}}$ values giving acceptable fits is preferable to simply constraining $R_{\infty}$. Measuring $R_{\infty}$ is generally satisfactory, however, when the observational data is not of the highest quality, and/or the purpose of the measurement is simply to check for consistency with the canonical NS mass and radius (a typical test of current observational data).

Applicability of any pure hydrogen-atmosphere model to a NS atmosphere requires that accretion is not continuing above the critical rate to keep metals present in the atmosphere, as that would alter the opacity of the atmosphere (Brown et al. 1998). The X-ray luminosity produced by the critical accretion rate is similar to the observed X-ray luminosity of X7. Thus, it remains conceivable that the inferred mass and radius of X7 are biased by the presence of metals, and thus extra opacity, in the NS atmosphere. However, the extraordinary stability of X7's X-ray flux, and its lack of accretion signatures such as a hard power-law spectral component, or features due to lines or edges, indicate that X7's X-ray emission is most likely produced by deep crustal heating rather than continued accretion.

\section{CONCLUSIONS}

We have computed new grids of hydrogen atmosphere models for neutron stars in quiescent LMXBs, accounting selfconsistently for electron thermal conduction, radiation force, self-irradiation of the NS, and variations in the NS surface gravity. The first three effects do not produce large changes, and our NSATMOS models agree very well with the NSA models of (Zavlin et al. 1996) in the appropriate surface gravity regime. We find that the effects of freely varying the NS surface gravity, on the other hand, are significant, especially when trying to constrain the mass and radius of neutron stars.

We report new (2002) Chandra observations of the quiescent LMXB known as X7 in 47 Tuc. No variability is observed on any time scale between minutes and weeks in the new data set. The 2002 Chandra data, plus the 2000 Chandra data, can be simultaneously fit with our hydrogen atmosphere model, photoelectric absorption, and a correction model for instrumental pileup. No convincing spectral features are seen, and no additional hard component is detected. No variations are seen in X7's spectrum over the 2.5 year interval.

Our spectral fitting constrains the range of mass and radius which can produce a spectrum like that observed. In contrast to the results of Heinke et al. (2003), our use of a range of surface gravities allows acceptable fits to X7 with a standard $1.4 M_{\odot}$ NS mass and a radius of $14.5_{-1.6}^{+1.8} \mathrm{~km}$, as well as a highmass solution $\left(\mathrm{M}=2.20_{-0.16}^{+0.03} M_{\odot}\right)$ for a radius of $10 \mathrm{~km}$. For a canonical mass of $1.4 M_{\odot}$ a $10-12 \mathrm{~km}$ radius is ruled out at $99 \%$ confidence. This indicates (assuming the validity of a pure hydrogen atmosphere model) that either $\mathrm{X} 7$ is more massive than any NS yet known, or that X7 has a somewhat larger radius than canonical modern NS models (our preferred interpretation). In either case a relatively "stiff" NS equation of state is favored.

The HYD_SPECTRA (Gänsicke et al. 2002) and NSA hydrogen-atmosphere NS models as currently implemented in XSPEC do not include a range of surface gravities appropriate for the possible ranges of NS masses and radii. Our work using NSATMOS has shown that accurate constraints (at the $<10 \%$ level) on the radius and mass of NSs require self-consistent modeling of the effects of surface gravity. Our NSATMOS model, and the NSAGRAV code (using the models of Zavlin et al. 1996, provided for XSPEC during this paper's refereeing process), meet these requirements.

We warmly acknowledge Don Lloyd for providing comparison neutron star atmosphere models for use in verifying our code, and Marc Freitag for the use of his fig2curve script. We also thank Lars Bildsten, Bob Rutledge and Jim Lattimer for useful discussions and suggestions, and the CXC team for their untiring data calibration efforts. This work was supported in part by NSF grant AST 0307433, and in part by the Lindheimer Postdoctoral Fellowship at Northwestern University.

\section{APPENDIX}

\section{THE NSATMOS CODE}

The NSATMOS code calculates an atmospheric model under the following conditions and assumptions: (1) Static, atmosphere in the plane-parallel approximation; (2) Negligible magnetic fields $\left(B \lesssim 10^{8} \mathrm{G}\right)$; (3) Ideal equation of state for pure hydrogen with complete ionization; (4) Opacity due to thermal free-free absorption plus Thomson scattering in the unpolarized, isotropic approximation; (5) Energy transport by radiation and electron heat conduction; (6) Hydrostatic equilibrium includes radiation force due to absorption and scattering; (7) Comptonization is ignored; and (8) For a compact object within its own photon sphere, $R<(3 / 2) R_{\mathrm{S}}$, self-irradiation of the surface is taken into account $\left(R_{\mathrm{S}}=2 G M / c^{2}\right.$ is the Schwarzschild radius). We note that the neglect of neutral hydrogen limits the validity of the code to temperatures $T_{\text {eff }} \gtrsim 3 \times 10^{5} \mathrm{~K}$, while the omission of Comptonization limits it to perhaps $T_{\text {eff }} \lesssim 3 \times 10^{6} \mathrm{~K}$ (Zavlin et al. 1996). These limitations are not serious for the present application, but work is in progress to correct NSATMOS for both.

The solution of the atmosphere problem based on these assumptions follows mostly along standard lines for stellar atmospheres. Here we review the main ideas for reference.

The depth variable used here is the mass column density $m$, measured from the surface, which is related to vertical height $z$ by the differential relation $d m=-\rho d z$, where $\rho$ is the mass density.

The intensity field $I_{\nu}(m, \mu)$ is a function of frequency $\nu$, depth, and $\mu$, the cosine of the angle relative to the outward normal.The transfer equation for the intensity is then

$$
\mu \frac{\partial I_{\nu}}{\partial m}=\kappa_{\nu}\left(I_{\nu}-B_{\nu}\right)+\kappa_{\mathrm{T}}\left(I_{\nu}-J_{\nu}\right)
$$

The Planck function is denoted by $B=B_{\nu}(T)$, where $T=T(m)$ is the local temperature. For a fully ionized hydrogen plasma the Thomson opacity $\kappa_{\mathrm{T}}$ is a constant, but the free-free opacity $\kappa_{\nu}$ depends on the density and temperature. The mean intensity $J_{\nu}$ is 
defined by the integral,

$$
\frac{1}{2} \int_{-1}^{+1} \mu^{j} I_{\nu} d \mu
$$

for $j=0$. Other useful moments $H_{\nu}$ and $K_{\nu}$ are defined by this integral for $j=1$ and $j=2$, respectively.

When the stellar surface is outside the photon sphere, $R>(3 / 2) R_{\mathrm{S}}$, the surface boundary condition on the intensity field $I_{\nu}(m, \mu)$ is the usual one of no incident radiation. When $R<(3 / 2) R_{\mathrm{S}}$, the self-irradiation of the surface due to gravitational bending of the rays is taken into account as discussed in McClintock et al. (2004). The boundary condition at suffiently large depth is that the radiation field is given by the LTE diffusion approximation based on the local gradient of the temperature field.

An important quantity is the radiative monochromatic energy flux, given in terms of the $H_{\nu}$ moment by $F_{\nu}=4 \pi H_{\nu}$. The total radiative energy flux is found by integration over frequency,

$$
F_{\text {rad }}=\int_{0}^{\infty} F_{\nu} d \nu=4 \pi \int_{0}^{\infty} H_{\nu} d \nu .
$$

For some ranges of parameters of interest, the heat conduction by electrons can be of importance. For the conductive flux we use the Spitzer-Härm formula

$$
F_{\text {con }}=-\lambda_{\mathrm{c}} T^{5 / 2} \frac{\partial T}{\partial z},
$$

The constant $\lambda_{\mathrm{c}}$ has a standard value of order $1.8 \times 10^{-5} \mathrm{erg} \mathrm{cm}^{-1} \mathrm{~s}^{-1} \mathrm{~K}^{-7 / 2}$. Magnetic fields tend to suppress the thermal conduction, though by only a factor of order 3-5 when the field is chaotically tangled (Naravan \& Medvedev 2001). Therefore, for the calculations of this paper we have assumed a conductivity equal to a third of the Spitzer value. However, we find that electron conduction even at the Spitzer value is a negligible contributor to the energy flux, making the issue moot. We take as a boundary condition at the surface that the conductive flux vanishes, $F_{\text {con }}(0)=0$, since no electrons exist above the outer surface.

Energy equilibrium requires that the net outward energy flux, radiative plus conductive, be constant with depth,

$$
F_{\text {tot }}=F_{\text {rad }}+F_{\text {con }}=\sigma T_{\text {eff }}^{4} \text {. }
$$

The constant total flux is parametrized by the effective temperature $T_{\mathrm{eff}}$. Since the conductive flux vanishes at the surface, the effective temperature is, as usual, a measure of the total emitted radiative flux.

Equation A5 is used in NSATMOS as the basic expression of energy equilibrium. We note that another common method of expressing this is through the flux derivative condition,

$$
\frac{d F_{\text {tot }}}{d m}=0
$$

If this form is used, the desired value of total flux $F_{\text {tot }}$ must be introduced in some other way, usually through the boundary conditions at depth on radiation and conduction.

The hydrostatic equilibrium equation is

$$
\frac{\partial p}{\partial m}=g-g_{\mathrm{rad}}
$$

where $p$ is the gas pressure and $g$ is the local acceleration of gravity, given by the usual formulas in Schwarzschild geometry. The radiative weakening of gravity $g_{\text {rad }}$ is given by the radiation force per unit mass due to free-free absorption and Thomson scattering,

$$
g_{\text {rad }}=\frac{1}{c} \int\left(\kappa_{\nu}+\kappa_{\mathrm{T}}\right) F_{\nu} d \nu .
$$

In a completely ionized hydrogen plasma, the electron and proton densities are equal, $n_{\mathrm{e}}=n_{\mathrm{p}}$, and the ideal equation of state is

$$
p=2 n_{\mathrm{e}} k T
$$

where $k$ is the Boltzmann constant.

The model atmosphere problem requires the simultaneous, self-consistent solution of the preceding equations for the radiation field and the gas properties (temperature, density, and pressure) as functions of depth. Overall, this is a set of nonlinear, coupled equations. Our method of solution involves (as do all such methods) a preliminary recasting of the equations into favorable forms such that cycles of iteration between them converge reasonably rapidly to the desired solution.

One very useful trick is to recast the transfer equation in terms of Eddington factors. Multiplication of equation A1 by 1 and $\mu$, followed by integration over all $\mu$ gives

$$
\begin{aligned}
& \frac{\partial H_{\nu}}{\partial \tau_{\nu}}=\epsilon_{\nu}\left(J_{\nu}-B_{\nu}\right) \\
& \frac{\partial K_{\nu}}{\partial \tau_{\nu}}=H_{\nu}
\end{aligned}
$$

where the monochromatic optical depth is defined differentially as $d \tau_{\nu}=\left(\kappa_{\nu}+\kappa_{\mathrm{T}}\right) d m$ with $\tau_{\nu}=0$ at the surface, and where

$$
\epsilon=\frac{\kappa_{\nu}}{\kappa_{\nu}+\kappa_{\mathrm{T}}}
$$


Introducing the Eddington factor $f_{\nu}=K_{\nu} / J_{\nu}$, equations (A10) and A11) can be expressed as the single second-order equation,

$$
\frac{\partial^{2}\left(f_{\nu} J_{\nu}\right)}{\partial \tau_{\nu}^{2}}=\epsilon_{\nu}\left(J_{\nu}-B_{\nu}\right)
$$

The two boundary conditions on this equation at the surface and at depth are expressed through the boundary Eddington factors $g_{\nu 0}$ and $g_{\nu d}$, such that,

$$
H_{\nu}(0)=g_{\nu 0} J_{\nu}(0), \quad H_{\nu}\left(\tau_{\nu d}\right)=g_{\nu d} J_{\nu}\left(\tau_{\nu d}\right) .
$$

After a set of initial Eddington factors is chosen, they are updated during the course of iterative solution using full angular formal solutions of the transfer equation (A1]. It is during this formal solution that the detailed boundary conditions on intensity come into play, including the possibility of sef-irradiation.

A particularly critical part of the iterative solution is how the run of temperature with depth $T(m)$ is corrected after each iteration. In doing this it is important to take account of how radiation can couple the variables at distant points in the atmosphere. Methods that try to correct the temperature based on local information, or even on information just a few optical depths away, will converge much too slowly to be practical.

Here we adopt a temperature correction scheme based on a partial linearization of the temperature dependence in the transfer equation and the energy equation. The temperature field is represented as

$$
T(m)=T^{(0)}(m)+T^{(1)}(m),
$$

where $T^{(0)}(m)$ is an initial "guess" for the temperature, and $T^{(1)}(m)$ is the "correction." In the perturbation sense these are regarded as of zeroth and first order, respectively. Other quantities in the problem can be expanded to first order in the perturbation. For example, the radiation field has the representation,

$$
I_{\nu}=I_{\nu}^{(0)}+I_{\nu}^{(1)}
$$

Not all quantities in the problem are expanded in this way, making this a partial linearization rather than a complete linearization scheme. The decision as to which quantities are expanded and which are not is somewhat arbitrary, but is guided by the physical idea that the critical equations are the energy equation, the transfer equation, and the conduction equation, since these are the ones that control the global redistribution of energy in the atmosphere.

The zeroth order form of the transfer equation $\mathrm{A13}$ is

$$
\frac{\partial^{2}\left(f_{\nu} J_{\nu}^{(0)}\right)}{\partial \tau_{\nu}^{2}}=\epsilon_{\nu}\left[J_{\nu}^{(0)}-B_{\nu}\left(T^{(0)}\right)\right] .
$$

We take as the first order form,

$$
\frac{\partial^{2}\left(f_{\nu} J_{\nu}^{(1)}\right)}{\partial \tau_{\nu}^{2}}=\epsilon_{\nu}\left[J_{\nu}^{(1)}-\dot{B}_{\nu}\left(T^{(0)}\right) T^{(1)}\right]
$$

where $\dot{B}(T)=\partial B_{\nu}(T) / \partial T$. Note that the radiation fields and the temperature dependence of the Planck function have been expanded, but not the Eddington factors or the opacities, so that the optical depth scale is unchanged.

Expansion of the energy equation A5 through first order gives, after some rearrangement,

$$
F_{\mathrm{tot}}^{(1)}=F_{\mathrm{rad}}^{(1)}+F_{\mathrm{con}}^{(1)}=\sigma T_{\mathrm{eff}}^{4}-\left(F_{\mathrm{rad}}^{(0)}+F_{\mathrm{con}}^{(0)}\right)=-E .
$$

The right hand side is the negative of the flux "error" $E$ in the zeroth order solution. The left hand side is the total first order flux, consisting of the two terms,

$$
\begin{aligned}
& F_{\text {rad }}^{(1)}=4 \pi \int_{0}^{\infty} H_{\nu}^{(1)} d \nu, \\
& F_{\text {con }}^{(1)}=-\lambda_{\mathrm{c}} \frac{\partial}{\partial z}\left[\left(T^{(0)}\right)^{5 / 2} T^{(1)}\right]
\end{aligned}
$$

Each of these depends linearly on the first order temperature correction $T^{(1)}$. For $F_{\mathrm{con}}^{(1)}$ this follows directly from equation A21. For $F_{\text {rad }}^{(1)}$ we note that $H^{(1)}$ depends linearly on $J_{\nu}^{(1)}$, since

$$
\frac{\partial\left(f_{\nu} J_{\nu}^{(1)}\right)}{\partial \tau_{\nu}}=H_{\nu}^{(1)}
$$

which follows from equation A11, noting $K_{\nu}^{(1)}=f_{\nu} J_{\nu}^{(1)}$. Finally, $J_{\nu}^{(1)}$ depends linearly on $T^{(1)}$ through equation A18

In terms of $N_{D}$ discretized depths, the linear dependence of $F_{\text {rad }}^{(1)}(m)$ and $F_{\text {con }}^{(1)}(m)$ on $T^{(1)}(m)$, can be expressed as

$$
\begin{aligned}
& F_{\text {rad }}^{(1)}=\Phi_{\text {rad }} \cdot T^{(1)}, \\
& F_{\text {con }}^{(1)}=\Phi_{\text {con }} \cdot T^{(1)},
\end{aligned}
$$

where $\Phi_{\text {rad }}$ and $\Phi_{\text {con }}$ are finite matrix operators over the discrete depths. 
In order to construct the matrix $\Phi_{\text {rad }}, N_{F}$ discrete frequencies are introduced. For each of these frequencies, the transfer equation A17, plus its boundary conditions, becomes a tridiagonal matrix equation in depth, which may be solved in of order $N_{D}^{2}$ operations. In this way one obtains matrices $A_{\nu}$ such that $J_{\nu}^{(1)}=A_{\nu} \cdot T^{(1)}$ for each frequency. Implementing equation (A22) with some form of numerical differentiation, we obtain matrices $B_{\nu}$ such that $F_{\nu}^{(1)}=B_{\nu} \cdot T^{(1)}$. Implementing a frequency quadrature scheme for equation $\mathrm{A} 20$, we then obtain the matrix $\Phi_{\text {rad. }}$. The whole process takes of order $N_{F} N_{D}^{2}$ operations.

The construction of the matrix $\Phi_{\text {con }}$ is easier, since it merely involves implementing a numerical differentiation formula for equation A21 plus boundary condition. Depending on the order of the formula, the resulting matrix might be bidiagonal or tridiagonal.

Defining $\Phi_{\text {tot }}=\Phi_{\text {rad }}+\Phi_{\text {con }}$, equations A19, A23, and A24 can be combined to give,

$$
\Phi_{\text {tot }} \cdot T^{(1)}=-E \text {, }
$$

which relates the temperature correction $T^{(1)}$ directly to the flux error $E$ in the trial (zeroth order) solution. This matrix equation is then solved for $T^{(1)}$, and a new trial temperature law is obtained from $T^{(0)} \leftarrow T^{(0)}+T^{(1)}$.

Using the new trial temperature, improved radiation fields, Eddington factors, radiative force, and hydrostatic equilibrium are computed. This process is repeated until convergence is obtained.

This method was used to compute our grid of models. In order to initialize the first model, we used a Rosseland-type solution for the run of temperature (similar to the asymptotic result given in equation (15) of Zavlin et al. 1996) and we set the radiative force to zero. For subsequent models we adopted those quantities from a previously calculated model with nearby parameters. This was particularly helpful in computing models close to the Eddington limit.

Some additional "tricks" we found useful for some models were: (1) Adiabatic turning on of parameters, in which the model parameters were changed in small steps from those of a previously solved case to the desired ones, these changes occuring along with the other iterations; and (2) Linearization limiters, which are virtually identical to A15 for small corrections, but which keep the temperature correction within fixed bounds, for example,

$$
T(m)=T^{(0)}(m)+\frac{T^{(0)}(m)}{2} \tanh \left[\frac{2 T^{(1)}(m)}{T^{(0)}(m)}\right] .
$$

\section{SURFACE GRAVITY EFFECTS}

Our spectral fitting to constrain X7 in the mass-radius plane has shown the importance of considering neutron star models with a full range of surface gravities as well as effective temperatures. This conclusion is based on numerical results of the XSPEC analysis program, which involves a long chain of data reductions plus manipulations of the the model atmosphere spectra. In this appendix we attempt to understand better the importance of the surface gravity $g_{\mathrm{s}}$ using some heuristic, qualitative arguments.

We begin by considering a approximate analytical representation for the surface spectral flux of the NS atmosphere, namely,

$$
F_{\nu}(\nu)=T_{\mathrm{eff}}{ }^{3} \phi(\xi), \quad \xi=\nu / T_{\mathrm{eff}},
$$

where $\phi(\xi)$ is a fixed function. This implies that there is a single spectral shape which is simply rescaled by the effective temperature $T_{\text {eff. }}$. Such a representation would hold exactly if the neutron star surface radiated as a blackbody. It also holds approximately for more realistic pure ionized hydrogen atmospheres; an approximate expression of this form was given in equation (A17) of McClintock et al. (2004).

Given the spectral flux at the surface, the observed spectral flux $F_{\text {obs }}\left(\nu_{\text {obs }}\right)$ at a large distance $D$ may be expressed (see, e.g., McClintock et al. 2004). Let us assume for the moment that the surface flux is given by (B1) exactly. Then for a neutron star with mass $M_{\mathrm{ns}}$ and radius $R_{\mathrm{ns}}$ we may write,

$$
F_{\mathrm{obs}}\left(\nu_{\mathrm{obs}}\right)=\frac{R_{\infty}^{2}}{D^{2}} T_{\infty}^{3} \phi\left(\nu_{\mathrm{obs}} / T_{\infty}\right),
$$

where $T_{\infty}=T_{\mathrm{eff}} /(1+z), R_{\infty}=R_{\mathrm{ns}}(1+z)$. Here $1+z=\left(1-R_{\mathrm{S}} / R_{\mathrm{ns}}\right)^{-1 / 2}$ is the gravitational redshift factor at the neutron star surface, and $R_{\mathrm{S}}=2 G M_{\mathrm{ns}} / c^{2}$ is the Schwarzschild radius.

With a prescribed form of the function $\phi$ and a known distance $D$, spectral fitting is reduced to the determination of just two parameters, $T_{\infty}$ and $R_{\infty}$. However, there are three parameters required to specify a neutron star model fully, which can be taken to be its mass $M_{\mathrm{ns}}$, radius $R_{\mathrm{ns}}$, and effective temperature $T$. This implies that for each fit there is a one-parameter set of models that are observationally indistinguishable from that fit. To show this in detail, we first note that the relation $R_{\infty}=R_{\mathrm{ns}}(1+z)$ can be expressed,

$$
M_{\mathrm{ns}}=\left(1-\frac{R_{\mathrm{ns}}^{2}}{R_{\infty}^{2}}\right) \frac{c^{2} R_{\mathrm{ns}}}{2 G} .
$$

An acceptable $M_{\mathrm{ns}}-R_{\mathrm{ns}}$ pair can lie anywhere on one of the contour lines of constant $R_{\infty}$, shown as the solid curves in figure B1 a). The additional parameter along each curve can be taken to be the ratio $T_{\text {eff }} / T_{\infty}$, which is equal to the redshift factor (1+z), given in terms of $R_{\mathrm{ns}}$ and $M_{\mathrm{ns}}$ by

$$
1+z=\left(1-\frac{2 G M_{\mathrm{ns}}}{c^{2} R_{\mathrm{ns}}}\right)^{-1 / 2} .
$$

Curves of constant $(1+z)=T_{\text {eff }} / T_{\infty}$ are plotted as dashed curves in figure B1 a). However, since there is no independent determination of $T_{\text {eff }}$, this relation does not constrain the possible locations along the contours of $R_{\infty}$. In a spectral fitting program such 

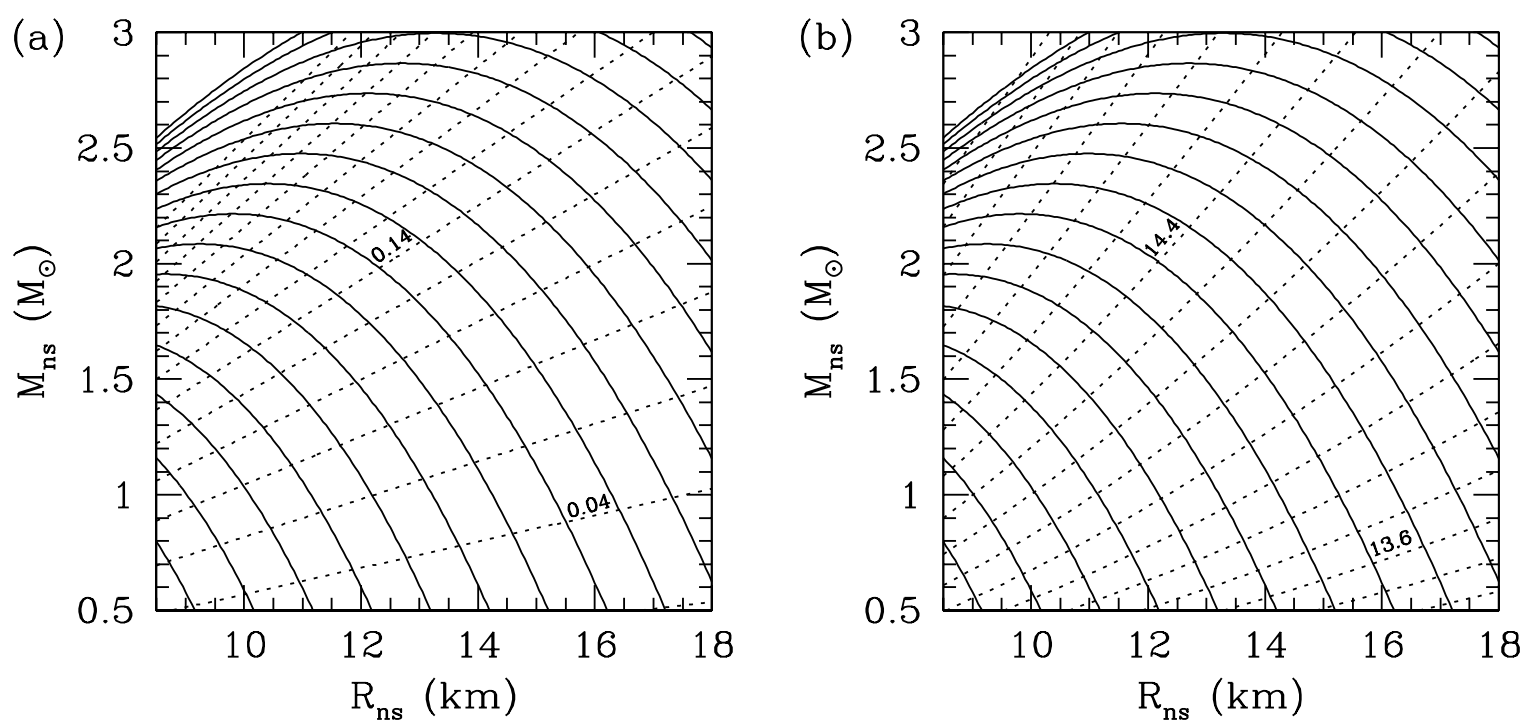

FIG. B 1. - (a) The $M_{\mathrm{ns}}-R_{\mathrm{ns}}$ plane, showing solid curves of constant $R_{\infty}$ and dashed curves of constant (1+z). Two of these dashed curves are labelled for the values $\log (1+z)=0.04$ and 0.14 . The others are at logarithmic spaced values with $\Delta \log (1+z)=0.02$. (b) The same, except with dashed curves of constant surface gravity $g_{\mathrm{s}}$. The two marked curves are for $\log g_{\mathrm{s}}=13.6$ and 14.4 , with other curves spaced with $\Delta \log g_{\mathrm{s}}=0.1$.
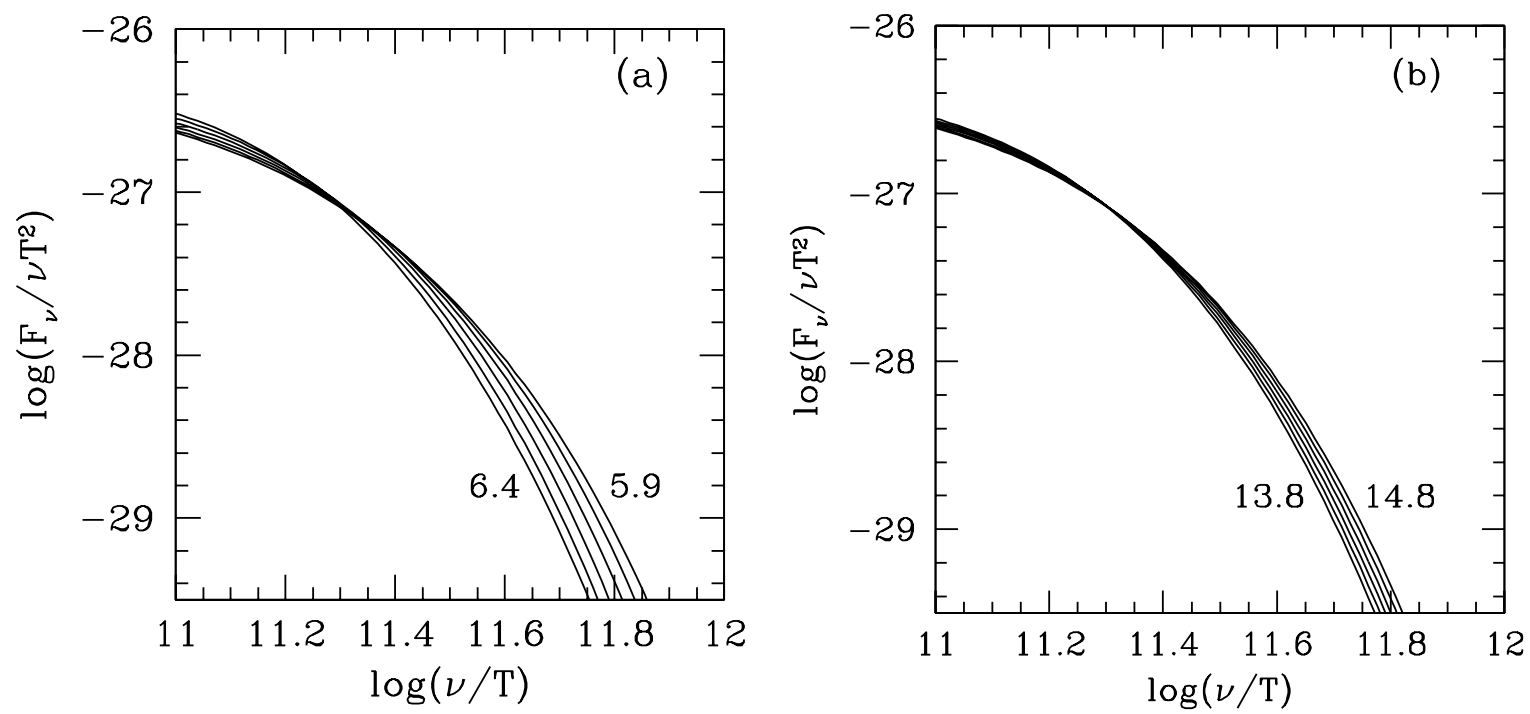

FIG. B2.- (a) Scaled surface number flux vs. scaled frequency for $\log g_{\mathrm{s}}=14.4$ and for $\log T_{\text {eff }}$ ranging from 5.9 to 6.4. (b) Scaled surface number flux vs. scaled frequency for $\log T_{\text {eff }}=6.2$ and for $\log g_{\mathrm{s}}$ ranging from 13.8 to 14.8 .

as XSPEC, this degeneracy would show itself by the fact that the confidence contours would be made up of contours of constant $R_{\infty}$, that is, the solid curves in figure $\mathrm{B} 1 \mathrm{a}$ ).

In practice, since the simple scaling law (B1) is only approximately valid, the confidence contours will not follow those solid curves precisely. Nonetheless, one can see in figures 2 3 and 4 that the confidence contours are generally consistent with there being a blackbody-like degeneracy along their length, since they do not close off on either ends of the confidence bands. One also sees that the shapes of the confidence contours are different depending on whether the gravity has been fixed or allowed to vary freely. The fixed gravity curves can be characterized as being pushed out to larger radii at smaller mass, giving the curves in this region a flatter appearance. The curves for freely varying gravity do the opposite: they are pushed to smaller radii at smaller mass, so much so that they actually curl around and change their direction. (It is this fact that allows a fit with a smaller radius, one of the major results of this paper.)

We can give a heuristic, qualitative argument for this difference. First, let us note that the surface gravity, which is required for the construction of the atmosphere, is given by,

$$
g_{\mathrm{s}}=\frac{G M_{\mathrm{ns}}(1+z)}{R_{\mathrm{ns}}^{2}}
$$

In figure B1 b), curves of constant $R_{\infty}$ (solid) are again plotted in the $M_{\mathrm{ns}}-R_{\mathrm{ns}}$ plane, but now with curves of constant $g_{\mathrm{s}}$ (dashed).

The sensitivity of the function $\phi\left(\nu / T_{\text {eff }}\right)$ to temperature and gravity is demonstrated in figure B2 Here is plotted the quantity $\log \left(F_{\nu} / \nu T_{\text {eff }}{ }^{2}\right)$ versus $\log \left(\nu / T_{\text {eff }}\right)$ for the surface fluxes of the neutron star models for various effective temperatures $[\bar{B} 2$ a $\left.a)\right]$ and 
various gravities [B2 $\mathrm{b}$ )] in the neighborhood of the approximate fitted values $\log T_{\text {eff }}=6.2$ and $\log g_{\mathrm{s}}=14.4$. The frequency range here was chosen to match roughly the portion of the flux curves observed in X7. If the scaling relation (B1) were exact, these would all lie on the same curve, namely, $\log [\phi(\xi) / \xi]$. In fact, there is a sensitivity to both effective temperature and gravity. We see that the flux distribution steepens for increasing temperature, and for decreasing surface gravity. The sensitivity to temperature is greater than that of gravity, in the sense that 0.5 dex variation of temperature gives a substantially greater variation than 1.0 dex variation in gravity. However, an essential point to notice from figures B1 a) and B1 b) is that over the part of the $M_{\mathrm{ns}}-R_{\mathrm{ns}}$ plane where the spectral fitting results are most divergent, say, for $0.5<M_{\mathrm{ns}} / M_{\odot}<2$ and for $12 \mathrm{~km}<R_{\mathrm{ns}}<18 \mathrm{~km}$, the gravity changes by about 1.0 dex, while the temperature changes by about only 0.1 dex [a range corresponding to two neighboring curves in figure B2 a)]. In that case, the change due to gravity is effectively more substantial than that due to temperature. Also, the changes in the curves are of the opposite sense as one moves along the curves of constant $R_{\infty}$ from the top to bottom of the plot, since both temperature and gravity decrease. Thus, if one fixes the gravity, the confidence contours are pushed in one way by the sensitivity to temperature, but if gravity is allowed to vary, then the larger effective sensitivity to gravity pushes the confidence curves in the opposite direction. The preceding heuristic argument accounts qualitatively for the overall behavior seen in figures 2,3 and 4 and demonstates the need to include variable gravity when doing spectral fitting for such objects.

\section{REFERENCES}

Akmal, A., Pandharipande, V. R., \& Ravenhall, D. G. 1998, Phys. Rev. C, 58, 1804

Arnaud, K. A. 1996, in ASP Conf. Ser. 101: Astronomical Data Analysis Software and Systems V, 17

Bogdanov, S., Grindlay, J. E., \& van den Berg, M. 2005, ApJ, 630, 1029

Broos, P., Townsley, L., Getman, K., \& Bauer, F. 2002, ACIS Extract, An ACIS Point Source Extraction Package, Pennsylvania State University, http://www.astro.psu.edu/xray/docs/TARA/ae_users_guide.html

Brown, E. F., Bildsten, L., \& Rutledge, R. E. 1998, ApJ, 504, L95

Burderi, L., Di Salvo, T., D’Antona, F., Robba, N. R., \& Testa, V. 2003, A\&A, 404, L43

Campana, S., Colpi, M., Mereghetti, S., Stella, L., \& Tavani, M. 1998, A\&A Rev., 8, 279

Campana, S., Israel, G. L., Stella, L., Gastaldello, F., \& Mereghetti, S. 2004 , ApJ, 601, 474

Campana, S., Stella, L., Gastaldello, F., Mereghetti, S., Colpi, M., Israel, G. L., Burderi, L., Di Salvo, T., \& Robba, R. N. 2002, ApJ, 575, L15

Cardelli, J. A., Clayton, G. C., \& Mathis, J. S. 1989, ApJ, 345, 245

Carney, B. W. 1996, PASP, 108, 900

Chandler, A. M., \& Rutledge, R. E. 2000, ApJ, 545, 1000

Colpi, M., Geppert, U., Page, D., \& Possenti, A. 2001, ApJ, 548, L175

Cottam, J., Paerels, F., \& Mendez, M. 2002, Nature, 420, 51

Davis, J. E. 2001, ApJ, 562, 575

Gänsicke, B. T., Braje, T. M., \& Romani, R. W. 2002, A\&A, 386, 1001

Garcia, M. R., McClintock, J. E., Narayan, R., Callanan, P., Barret, D., \& Murray, S. S. 2001, ApJ, 553, L47

Gendre, B., Barret, D., \& Webb, N. 2003a, A\&A, 403, L11

Gendre, B., Barret, D., \& Webb, N. A. 2003b, A\&A, 400, 521

Gratton, R. G., Bragaglia, A., Carretta, E., \& et al. 2003, A\&A, 408, 529

Grindlay, J. E., Heinke, C., Edmonds, P. D., \& Murray, S. S. 2001, Science, 292,2290

Heinke, C. O., Grindlay, J. E., Edmonds, P. D., Cohn, H. N., Lugger, P. M., Camilo, F., Bogdanov, S., \& Freire, P. C. 2005, ApJ, 625, 796

Heinke, C. O., Grindlay, J. E., Lloyd, D. A., \& Edmonds, P. D. 2003, ApJ, 588,452

Lattimer, J. M., \& Prakash, M. 2001, ApJ, 550, 426

Leahy, D. A., Darbro, W., Elsner, R. F., Weisskopf, M. C., Kahn, S., Sutherland, P. G., \& Grindlay, J. E. 1983, ApJ, 266, 160

Lloyd, D. A. 2003, Ph.D. Thesis
McClintock, J. E., Narayan, R., \& Rybicki, G. B. 2004, ApJ, 615, 402

Müller, H., \& Serot, B. D. 1996, Nuclear Physics A, 606, 508

Narayan, R., \& Medvedev, M. V. 2001, ApJ, 562, L129

Nice, D. J., Splaver, E. M., Stairs, I. H., Löhmer, O., Jessner, A., Kramer, M., \& Cordes, J. M. 2005, ApJ, 634, 1242

Olson, T. S. 2001, Phys. Rev. C, 63, 015802

Pandharipande, V. R., \& Ravenhall, D. G. 1989, in NATO ASIB Proc. 205: Nuclear Matter and Heavy Ion Collisions, 103-+

Pavlov, G. G., Shibanov, I. A., \& Zavlin, V. E. 1991, MNRAS, 253, 193

Pons, J. A., Walter, F. M., Lattimer, J. M., Prakash, M., Neuhäuser, R., \& An, P. 2002, ApJ, 564, 981

Prakash, M., Cooke, J. R., \& Lattimer, J. M. 1995, Phys. Rev. D, 52, 661

Predehl, P., \& Schmitt, J. H. M. M. 1995, A\&A, 293, 889

Rajagopal, M., \& Romani, R. W. 1996, ApJ, 461, 327

Romani, R. W. 1987, ApJ, 313, 718

Rutledge, R. E., Bildsten, L., Brown, E. F., Pavlov, G. G., \& Zavlin, V. E. 1999, ApJ, 514, 945

-. 2001a, ApJ, 559, 1054

-. 2001b, АpJ, 551, 921

-. 2002a, ApJ, 578, 405

-. 2002b, ApJ, 577, 346

Salaris, M., \& Weiss, A. 1998, A\&A, 335, 943

Sanders, J. S., Fabian, A. C., Allen, S. W., \& Schmidt, R. W. 2004, MNRAS, 349,952

Stage, M. D., Joss, P. C., Madej, J., \& Różańska, A. 2004, Advances in Space Research, 33, 605

Thorsett, S. E., \& Chakrabarty, D. 1999, ApJ, 512, 288

Walter, F. M., \& Lattimer, J. M. 2002, ApJ, 576, L145

Wijnands, R., Heinke, C. O., Pooley, D., Edmonds, P. D., Lewin, W. H. G., Grindlay, J. E., Jonker, P. G., \& Miller, J. M. 2005a, ApJ, 618, 883

Wijnands, R., Homan, J., Heinke, C. O., Miller, J. M., \& Lewin, W. H. G. 2005b, ApJ, 619, 492

Wilms, J., Allen, A., \& McCray, R. 2000, ApJ, 542, 914

Yakovlev, D. G., \& Pethick, C. J. 2004, ARA\&A, 42, 169

Zampieri, L., Turolla, R., Zane, S., \& Treves, A. 1995, ApJ, 439, 849

Zavlin, V. E., Pavlov, G. G., \& Shibanov, Y. A. 1996, A\&A, 315, 141

Zavlin, V. E., Pavlov, G. G., \& Trumper, J. 1998, A\&A, 331, 821 